\title{
A-9. Angiographic Changes of the Veins of the Posterior Cranial Fossa in the Gerebellopontine Angle Tumors
}

\author{
Norihiko Tamaki, Hideyuki Yamashita \\ Kiyoshi Fujiwara and Seiya Shirakata \\ Department of Neurological Surgery, Kobe University \\ School of Medicine \\ Masato Kuninaka, Yoshiyuki Miyagi \\ Department of Anatomy, Kobe University \\ School of Medicine
}

Introduction: Displacement of the cerebellar arteries of the cerebellopontine angle tumor is well known and have been reported by many authors. Little attention have been paid, however, to the venous changes of the posterior cranial fossa. We have studied the topographic anatomy of the venous system of the posterior cranial fossa and reported the phlebographic signs of the cerebellopontine angle tumors.

Materials and Methods: For the study of the topographic anatomy, polyester plastic models of the veins of the posterior cranial fossa were made, using the cadavers ten to forty hours after death. Both carotid and vertebral ateries were canulated and flushed with two to three liters of physiological saline. The bilateral internal jugular veins were then canulated, and one and a half liters of polyester plastic, colored blue, were injected manually. Before injection of the plastic a tourniquet around the neck below the point of injection was applied. The whole body was kept in formalin overnight. The following day of injection the cranium was opened by supratentorial circular craniotomy and arcuate suboccipital craniotomy and the entire brain including the dura was removed. The dura and sinuses were preserved on the surface of the brain The brain removed from the cranium was fixed in 10 percent formalin for two to twelve weeks. After fixation the dura, arteries and pia-arachnoid were prepared, leaving the venous trees.

The veins of the mesencephalon and cerebellum were then anatomically studied. Ten cases of the cerebellopontine angle tumors were then studied, comparing the phlebograms of the vertebral angiography with the operative findings.

Displacement of the veins of the posterior cranial fossa caused by the tumor itself, by deformity of the brain stem, and by tonsillar herniation, in considering the size of the tumors, were described in detail.

Results: This method of plastic venous injection was very valuable for studying the topographic anatomy of veins of the posterior cranial fossa. The veins of the posterior cranial fossa were divided into three major groups, anterior, superior, and inferior group. The anterior group, of which the petorosal vein was the most prominant vein, drains the anterior portion of the brainstem and cerebellum and empties mainly into the superior and inferior petrosal sinuses. 
The superior group, which is composed of posterior mesencephalic vein, lateral mesencephalic vein, precentral vein and its tributeries, and superior vermian vein, drains the brain stem and superior and anterior portion of the mid-cerebellum.

They empties into the great cerebral vein of Galen and straight sinus. The inferior group, composed of inferior vermian vein and cerebellar hemispheric vein, drains mainly posterior and inferior portion of the cerebellum and primarily empties into the confluence, transverse sinus or tentorial sinus.

In case of the smaller size of the cerebellopontine angle tumor, for instance chest-nut size, there were only the lateral or upward displacement of the petrosal vein. Other veins were normal. In case of the larger size, for instance hens egg size of the cerebellopontine angle tumor, the petrosal veins and its tributaries were markedly displaced upward, upward and laterally, and dilated and stretched in angular or arcuate fashion. In other cases, avascular area was identified in the cerebellopontine angle region because of non-visualization of the petrosal vein, its tributaried and superior petrosal sinus. In case of presence of the deformity of the mesencephalon, the posterior mesencephalic veins, lateral mesencephalic veins, and branchial veins were suppressed medially and upward, showing the asymmetric cofiguration of them in anteroposterior view. Anterior ponto-mesencephalic vein was also displaced backward in lateral view.

The precentral vein was displaced backward and upward, the branchial tributaries on both side were separated, superior vermian vein was elevated, and the inferior vermian vein was displaced contralaterally. In case of tentorial herniation, tonsillo-vermian point of the inferior vermian vein was displaced backward and downward in lateral view, and inferior retrotonsillar vein was then stretched downward in antero-posterior view.

The degree of the displacement of the veins of the posterior cranial fossa depends upon the size of the tumor.

Conclusions: The topographic anatomy of the veins of the posterior cranial fossa and phlebographic signs of the cerebellopontine angle tumors were described in detail. Very valuable and detail informations on the pathologic changes of the cerebellopontine angle tumors could be obtained by anatomico-neuroradiological study of the veins of the posterior cranial fossa. The veins of the posterior cranial fossa are then of great diagostic significance in association with cerebellopontine angle tumors.

\section{REFERENCES}

1) Bradac, G. B.: The ponto-mesencephalic veins (Radio-anatomical study). Neuroradiology, 1, 52-57, 1970.

2) Bull, J. and Kozlowski, P.: The angiographic pattern of the petrosal veins in the normal and pathological. Neuroradiology, 1, 20-26, 1970.

3) Huang, Y. P. and Wolf, B. S.: The veins of the posterior fossa superior or Galenic draining group. Amer. J. Roentgenol., 95, 808-821, 1965.

4) Huang, Y. P. and Wolf, B. S.: Precentral cerebellar vein in angiography. Acta radiol., 5, 250-262, 1966.

5) Huang, Y. P., Wolf, B. S. and Okudera, T.: Angiographic anatomy of the inferior 
vermian vein of the cerebellum. Acta radiol., 9, 327-344, 1969.

6) Huang, Y. P., Wolf, B. S., Antin, S. P. and Okudera, T.: The veins of the posterior fossa-anterior or petrosal draining group. Amer. J. Roentgenol, Rad. Therapy and Nucl. Medicine. 104, 36-56, 1968.

7) Mine, T.: The medial inferior cerebellar vein. Brain and Nerve, 23, 129-141, 1971.

8) Mine, T.: The medial inferior cerebellar vein. Brain and Nerve 23, 639-649, 1971.

9) Mizukami, M. and Mine, T.: Diagnostic value of the precentral cerebellar vein in cases of the posterior fossa tumors. Brain and Nerve, 21, 117-122, 1969.

10) Takahashi, M., Wilson, G. and Hanafee, W.: The significance of the petrosal vein in the diagnosis of cerebellopontine angle tumors. Radiology, 89, 834-840, 1967.

\title{
A-10. Three Gases of Stationary Wave in Internal Carotid Artery
}

\author{
Shuzo Okumura, Koji Sato and Shoji Fujita \\ Neurosurgical Service of National Hospital in Okayama \\ Toshihiko Mryamoto \\ Department of Neurological Surgery, Okayama University, Medical School
}

We presented 3 cases of stationary wave in internal carotid artery, those were seen in the 3,000 consecutive series of carotid angiogram, which was obtained by perctaneous puncture of common carotid artery.

Case 1, 24 year-old female had been repeated right carotid angiograms 4 times to follow up the minor A-V malformation in her right frontal lobe. Follow-up duration was about a year. Stationary wave was seen at the 2 nd and 3 rd examination but in different figure at each examination, moreover, at each phase of serial angiogram of the each examination the figure appeared fluctuated. No abnormal findings was observed in the angiograms of the vertebral artery and abdominal aorta and its branches, especially renal arteries.

Case $2 ; 30$ year-old male suffered from acute onset right hemiplegia anda phasia. His left carotid angiogram showed stationary wave at the extracranial part and occulsion of the middle cerebral artery.

Case $3 ; 15$ year-old female, complained of repeated headache, has underwent left carotid angiogram which showed stationary wave but no pathological sign in the intracranial part.

The mechanism of the stationary wave will be explained by the spastic theory not conflicting with unstability of the wave figure. 